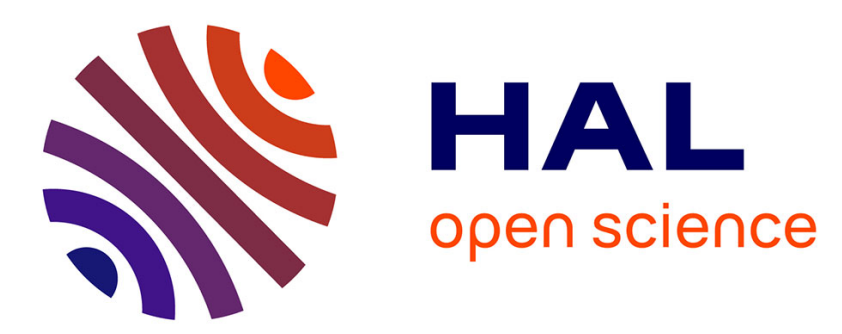

\title{
Input Independent Chaos Synchronization of Switched Systems
}

Gilles Millérioux, Jamal Daafouz

\section{To cite this version:}

Gilles Millérioux, Jamal Daafouz. Input Independent Chaos Synchronization of Switched Systems. IEEE Transactions on Automatic Control, 2004, 49 (7), pp.1182-1186. 10.1109/TAC.2004.831118 . hal-00121237

\section{HAL Id: hal-00121237 \\ https://hal.science/hal-00121237}

Submitted on 19 Dec 2006

HAL is a multi-disciplinary open access archive for the deposit and dissemination of scientific research documents, whether they are published or not. The documents may come from teaching and research institutions in France or abroad, or from public or private research centers.
L'archive ouverte pluridisciplinaire HAL, est destinée au dépôt et à la diffusion de documents scientifiques de niveau recherche, publiés ou non, émanant des établissements d'enseignement et de recherche français ou étrangers, des laboratoires publics ou privés. 


\title{
switched systems
}

\author{
Gilles Millerioux, Jamal Daafouz
}

\begin{abstract}
Input independent chaos synchronization of switched systems is addressed. The synchronization is carried out by designing an observer which involves a switched matrix gain. A special structure of this gain enables both an input independence of the state reconstruction error equation and a global convergence of this equation. Input independence is ensured by a suitable partial pole placement while the global convergence is based on the poly-quadratic stability concept. Those theoretical results would be interesting for possible applications in communications.
\end{abstract}

\section{INTRODUCTION}

For a large decade, since the pioneering works of [1], synchronization of chaotic motions has attracted a growing attention from the control theory point of view. One of the most popular approach for achieving the synchronization is based upon the framework of nonlinear observers. Indeed, synchronization problem has been shown [2] to be strongly related to the construction of the full state vector of the chaotic system from which only a transmitted signal consisting of a vector of smaller dimension is available. Distinct methodologies for the design of an observer have been examined. For relative recent results, the reader can refer to the "dynamic inversion" in the sense of [3], the concept of absolute stability [4], the observer design for chaotic systems with Generalized Hamiltonian Forms [5], the polytopic observers design based upon Linear Matrix Inequalities [6]. An attempt to mention all related papers on chaos synchronization would result in a prohibitively long list. It might be worthwhile to consult all devoted special issues and references therein appeared for that purpose [7][8][9][10].

Synchronization problems has become popular because of its possible use in communication. A huge number of papers has been published from this perspective, but security and performances of the proposed algorithms is a current research topic. Recent works and a serious overview can be found in [11] and references therein. What distinguishes the pure synchronization problem from the chaos-based communication problem is the fact that in the latter situation, an input,

Gilles Millerioux works in CRAN - ESSTIN (CNRS UMR 7039) 2, Rue Jean Lamour, 54519 Vandoeuvre-Les-Nancy (France) Email: millerioux@esstin.uhp-nancy.fr

Jamal Daafouz works in CRAN - ENSEM (CNRS UMR 7039) 2, Avenue de la Foret de Haye, 54516 Vandoeuvre-Les-Nancy (France) E-mail: jdaafouz@ensem.inpl-nancy.fr 
playing the role of the information to be masked, is embedded in the chaos generator, namely the drive. And yet, the input must be recovered at the so-called response side despite of the fact that it is not transmitted. To face this situation, two methods can be found in the existing literature. The problem is likely to be tackled from the identification point of view. The information is interpreted as a time-varying parameter, not available beforehand [12] and which must be identified. The second approach is based on nonlinear adaptive methods [13]. Both methods suffers from the fact that the parameter must be slowly time-varying to avoid too long convergence transients.

In this paper, a novel observer-based synchronization method is presented. It is called input independent global synchronization (IIGS) and enables to remove such a constraint for a special drive-response setup. The method first involves a partial pole placement technique which makes the equation governing the state reconstruction error to become input

independent. Partial pole placement has been widely investigated for linear systems. It consists of assigning a set of given eigenvalues while preserving stability by a suitable choice concerning the rest of the spectrum. In [14], a technique using dynamic output-feedback is presented. In [15], the problem is tackled while guaranteeing stability under LQ criteria. On the other hand, to date, very few papers, to our knowledge, have focus on partial pole for piecewise linear systems. Secondly, a special Lyapunov approach ensures the global convergence of the state reconstruction error. It is shown how this approach, resorting to poly-quadratic stability [16][17], turns the design of the gain matrices of the observer into a resolution of a set of Linear Matrix Inequalities.

The layout of the paper is as follows. Section II introduces the required formalism to establish the input independent state reconstruction problem as a special observer design. It is also devoted to some preliminary results required for the forthcoming sections. It particularly includes the poly-quadratic stability notion and the required structure of the observer gain so that the matrix governing the state reconstruction error gets a null space. Indeed, it will be shown that an input independent convergence property requires such a null space. In section III, the main result of the paper providing the design procedure to ensure input independent global convergence and the recovering of the original information is established. The switched matrix gain of the observer is derived from the solution of a set of Linear Matrix Inequalities. Finally, in order to assess the IIGS, an illustrative example borrowed from a communication context is given in section IV.

\section{Notations}

Throughout the paper, $\mathbf{1}$ (resp. 0) is an identity (resp. null) matrix of appropriate size. For a matrix $X, X^{T}$ stands for the transpose of $X, \mathcal{N}(X)$ is the null space of $X$. When being symmetric, $X>0$ indicates that $X$ is positive definite and the symbol $(\bullet)^{T}$ denotes each of its symmetric block. 
Consider the discrete time system :

$$
\begin{aligned}
& x_{k+1}=A_{i} \cdot\left(x_{k}+M_{i} u_{k}\right)+E_{i} \\
& y_{k}=C_{i} \cdot\left(x_{k}+M_{i} u_{k}\right)
\end{aligned}
$$

where $x_{k} \in \mathbb{R}^{n}, y_{k} \in \mathbb{R}^{m}, u_{k} \in \mathbb{R}^{m}$ are respectively the state vector, the output and the external input representing the information to be masked. The state space $x_{k} \in \mathbb{R}^{n}$ is partitioned into $P$ distinct regions $R_{i}$ with $\bigcup_{i=1}^{i=P} R_{i} \subseteq \mathbb{R}^{n}$. $A_{i}, M_{i}, E_{i}$ and $C_{i}$ are constant matrices assigned, with a one-to-one correspondence, to the region $R_{i}$ visited by $x_{k}$ at the discrete time $k$. The matrices $C_{i}$ are of rank $m, A_{i}$ and $E_{i}$ govern the dynamics such that a chaotic motion is exhibited. Finally, $M_{i}$ characterizes the way to mix the input $u_{k}$ with the chaotic signal $x_{k}$.

Remark 1. It is pointed out that for switched systems, chaos behaviors depend not only on the local dynamics but also on the rule which orchestrates the switches. One of the most popular piecewise linear systems are the Chua's circuit in the continuous time case, or the Tent Map, the Uniform Markov Map, the Logistic Map in the discrete time case.

Consider the discrete time system described by :

$$
\begin{aligned}
& \hat{x}_{k+1}=A_{i} \hat{x}_{k}+E_{i}+L_{i}\left(y_{k}-\hat{y}_{k}\right) \\
& \hat{y}_{k}=C_{i} \hat{x}_{k}
\end{aligned}
$$

This system acts as an observer with matrix gain $L_{i}$. The gain depends on the index $i$ since switched systems are considered. Throughout the paper, according to usual chaos synchronization terminology, the system (1) will be called drive and the system (2) will be refer to as response.

Problem is to design a drive-response system such that $\hat{x}_{k}$ tends asymptotically towards $x_{k}$ for any $u_{k}$, not available at the response side, and for any initial state $\hat{x}_{0}$. It amounts to find suitable matrices pairs $\left(M_{i}, L_{i}\right)$ for $i=1, \ldots, P$ such that:

$$
\lim _{k \rightarrow \infty}\left\|x_{k}-\hat{x}_{k}\right\|=0 \quad \forall \hat{x}_{0} \text { and } \forall u_{k}
$$

When (3) is verified, the drive-response system will be referred to as Input Independent Global Synchronized (IIGS). Before dealing with the main result of the paper, preliminary results are presented in next section. 
Consider the dynamical discrete time system which depends in a polytopic way on a vector $\xi_{k}$ :

$$
z_{k+1}=\mathcal{A}\left(\xi_{k}\right) z_{k}
$$

The structure of the dynamical matrix $\mathcal{A}$ gets the following polytopic description :

$$
\mathcal{A}\left(\xi_{k}\right)=\sum_{p=1}^{P} \xi_{k}^{p} A_{p}, \quad \xi_{k}^{p} \geq 0, \quad \sum_{p=1}^{P} \xi_{k}^{p}=1
$$

Following reasonings developed in [16], we consider Parameter-Dependent Lyapunov Functions (PDLF) of the form:

$$
V\left(z_{k}, \xi_{k}\right)=z_{k}^{T} \mathcal{P}\left(\xi_{k}\right) z_{k}
$$

with

$$
\mathcal{P}\left(\xi_{k}\right)=\sum_{p=1}^{P} \xi_{k}^{p} P_{p}
$$

the various $P_{p}, p=1, \ldots, P$, are symmetric positive definite matrices of appropriate order. The poly-quadratic stability concept is recalled in the following definition.

Definition 1. System (4) is said to be poly-quadratically stable if there exists a Positive Definite Parameter Dependent Quadratic Lyapunov Function (6) whose difference along the solution of (4) satisfies

$$
\mathcal{L}=V\left(z_{k+1}, \xi_{k+1}\right)-V\left(z_{k}, \xi_{k}\right) \leq-\alpha_{0}\left(\left\|z_{k}\right\|\right)
$$

with $\alpha_{0}$ a $\kappa_{\infty}$ function $^{1}$.

Poly-quadratic stability is sufficient for asymptotic stability. Assessing the poly-quadratic stability of a polytopic model is equivalent to find $P$ Positive Definite matrices $P_{p}, p=1, \ldots P$ such that :

$$
-\mathcal{P}\left(\xi_{k}\right)+\mathcal{A}^{T}\left(\xi_{k}\right) \mathcal{P}_{+}\left(\xi_{k}\right) \mathcal{A}\left(\xi_{k}\right)<0 \quad \forall k
$$

with :

$$
\left\{\begin{array}{l}
\mathcal{P}\left(\xi_{k}\right)=\sum_{p=1}^{P} \xi_{k}^{p} P_{p} \\
\mathcal{P}_{+}\left(\xi_{k}\right)=\sum_{p=1}^{P} \xi_{k+1}^{p} P_{p}
\end{array} \forall k\right.
$$

\footnotetext{
${ }^{1} \mathrm{~A}$ function $\alpha:[0, \infty) \rightarrow[0, \infty)$ is a $\kappa_{\infty}$ function if it is continuous, strictly increasing, zero at zero and unbounded $(\alpha(s) \rightarrow \infty$ as $s \rightarrow \infty)$.
} 
The following Theorem gives a necessary and sufficient condition for a time varying polytopic system such as (4) to be poly-quadratically stable.

Theorem 1. System (4) is poly-quadratically stable if and only if there exist symmetric positive definite matrices $S_{i}$ and matrices $G_{i}$ of appropriate dimensions such that

$$
\left[\begin{array}{cc}
G_{i}+G_{i}^{T}-S_{i} & (\bullet)^{T} \\
A_{i} G_{i} & S_{j}
\end{array}\right]>\mathbf{0}
$$

$\forall(i, j) \in\{1, \ldots, P\} \times\{1, \ldots, P\}$. In this case, the time varying PDLF is given by (6) with

$$
\mathcal{P}\left(\xi_{k}\right)=\sum_{p=1}^{P} \xi_{k}^{p} S_{p}^{-1} .
$$

Proof see [16].

Poly-quadratic stability is less conservative than quadratic stability which corresponds to the special choice of the Lyapunov function, $V\left(z_{k}, \xi_{k}\right)=z_{k}^{T} \mathcal{P}\left(\xi_{k}\right) z_{k}$ with $\mathcal{P}\left(\xi_{k}\right)=P^{*}$, a constant matrix, and thus corresponds to the particular case $G_{i}=S_{i}=S^{*}$, a constant matrix too. As far as piecewise linear systems are concerned, Theorem 1 holds by defining the quantity $\xi_{k}$ as an "indicator vector" given by the following definition :

Definition 2. An indicator vector associated to a switched system is a vector $\xi_{k}=\left(\xi_{k}^{1}, \ldots, \xi_{k}^{P}\right)^{T}$ whose components are defined as :

$$
\xi_{k}^{p}=\left\{\begin{array}{l}
1 \text { if } z_{k} \text { visits } R_{p} \\
0 \text { otherwise }
\end{array}\right.
$$

B. Stability under piecewise linear similarity transform

Consider the autonomous discrete time system :

$$
\eta_{k+1}=\Phi_{i} \eta_{k}
$$

where $\Phi_{i}$ belongs to a finite set of $P$ matrices. Let introduce a full rank piecewise linear transform $T_{i}$ and consider the recursion :

$$
\tilde{\eta}_{k+1}=T_{i}^{-1} \Phi_{i} T_{i} \tilde{\eta}_{k}
$$


where the succession of indexes $i$ and the discrete times $k$ corresponding to switches are the same as the ones of (12).

It can be stated that, the convergence towards zero of the solution $\tilde{\eta}^{*}$ of $(13)$ is equivalent the convergence towards zero of the solution $\eta^{*}$ of $(12)$.

Indeed, (13) can be rewritten :

$$
T_{i} \tilde{\eta}_{k+1}=\Phi_{i} T_{i} \tilde{\eta}_{k}
$$

which is equivalent to (12) and means that the samples $\eta_{k}^{*}$ and $\tilde{\eta}_{k}^{*}$ of the respective discrete trajectories $\eta^{*}$ and $\tilde{\eta}^{*}$ verify $\eta_{k}^{*}=T_{i} \tilde{\eta}_{k}^{*} . T_{i}$ being of full rank, since $\tilde{\eta}^{*}$ converges towards zero, it does the same for $\eta^{*}$.

\section{Input independence property}

In this subsection, a relation between $M_{i}$ and $L_{i}$ is stated in order to ensure the convergence of the system governing the state reconstruction error $\epsilon_{k}=x_{k}-\hat{x}_{k}$. This equation is obtained by subtracting (1) and (2):

$$
\epsilon_{k+1}=\left(A_{i}-L_{i} C_{i}\right) \epsilon_{k}+\left(A_{i}-L_{i} C_{i}\right) M_{i} u_{k}
$$

Proposition 1 For $i=1, \ldots, P$, assume that $L_{i}$ satisfies $\mathcal{N}\left(A_{i}-L_{i} C_{i}\right) \neq \emptyset$. Denote $N_{i}$ the matrix whose columns span the null space of $A_{i}-L_{i} C_{i}$. If $M_{i}=N_{i}$, thus the convergence is input independent, that is

$$
\epsilon_{k+1}=\left(A_{i}-L_{i} C_{i}\right) \epsilon_{k}
$$

Proof The proof is the immediate consequence of the definition of the null space. Indeed, for $i=1, \ldots, P,\left(A_{i}-\right.$ $\left.L_{i} C_{i}\right) M_{i}=\left(A_{i}-L_{i} C_{i}\right) N_{i}=\mathbf{0}$. Hence, (15) turns into (16).

\section{INPUT INDEPENDENT GLOBAL SYNCHRONIZATION}

This section is devoted to the design of the matrices $L_{i}$ and $M_{i}$ such that the drive-response system is both input independent as well as global synchronized.

\section{A. Input independence and partial pole placement}

In this subsection, a special form of the gains $L_{i}$ which guarantees that $A_{i}-L_{i} C_{i}$ has a non empty null space, required for input independence property, is presented. It is shown that the gains achieve a partial pole placement with zero 
eigenvalues.

Consider the following canonical equation :

$$
\tilde{\epsilon}_{k+1}=\left(\tilde{A}_{i}-\tilde{L}_{i} \tilde{C}_{i}\right) \tilde{\epsilon}_{k}
$$

where, for $i=1, \ldots, P, \tilde{A}_{i}=\left(T_{i}^{c}\right)^{-1} A_{i} T_{i}^{c}, \tilde{L}_{i}=\left(T_{i}^{c}\right)^{-1} L_{i}$ and $\tilde{C}_{i}=C_{i} T_{i}^{c}$ with $T_{i}^{c}$ the piecewise linear transform verifying :

$$
C_{i} T_{i}^{c}=\left[\begin{array}{ll}
\mathbf{0} & \mathbf{1}
\end{array}\right]=\tilde{C}_{i}
$$

Here, $\mathbf{0}$ is a $m \times(n-m)$ null matrix and $\mathbf{1}$ is the identity matrix of dimension $m . T_{i}^{c}$ always exists since $\operatorname{rank}\left(C_{i}\right)=m$.

Proposition 2 For $i=1, \ldots, P$, the gain $\tilde{L}_{i}=\tilde{A}_{i} \tilde{K}_{i}$ with $\tilde{K}_{i}=\left[\begin{array}{ll}\tilde{K}_{i}^{1} & \mathbf{1}\end{array}\right]^{T}$ ensures $\mathcal{N}\left(A_{i}-L_{i} C_{i}\right) \neq \emptyset$

Proof The proof is constructive in the sense that it provides the dimensionality of the null space. Consider the partitioned matrix $\tilde{A}_{i}$ with blocks of appropriate sizes :

$$
\tilde{A}_{i}=\left[\begin{array}{cc}
\tilde{A}_{i}^{11} & \tilde{A}_{i}^{12} \\
\tilde{A}_{i}^{21} & \tilde{A}_{i}^{22}
\end{array}\right]
$$

By direct calculation, one obtains :

$$
\tilde{A}_{i}-\tilde{L}_{i} \tilde{C}_{i}=\left[\begin{array}{cc}
\tilde{A}_{i}^{11} & -\tilde{A}_{i}^{11} \tilde{K}_{i}^{1} \\
\tilde{A}_{i}^{21} & -\tilde{A}_{i}^{21} \tilde{K}_{i}^{1}
\end{array}\right]
$$

Now, take the piecewise linear transform :

$$
T_{i}^{L}=\left[\begin{array}{cc}
\mathbf{1} & -\tilde{K}_{i}^{1} \\
\mathbf{0} & \mathbf{1}
\end{array}\right]
$$

Premultiplying (19) by the inverse of $T_{i}^{L}$ and postmultiplying by $T_{i}^{L}$, we obtain :

$$
\left(T_{i}^{L}\right)^{-1}\left(\tilde{A}_{i}-\tilde{L}_{i} \tilde{C}_{i}\right) T_{i}^{L}=\left[\begin{array}{cc}
\tilde{A}_{i}^{11}-\tilde{K}_{i}^{1} \tilde{A}_{i}^{21} & 0 \\
\tilde{A}_{i}^{21} & \mathbf{0}
\end{array}\right]
$$

Consequently, $\tilde{A}_{i}-\tilde{L}_{i} \tilde{C}_{i}$ and so $A_{i}-L_{i} C_{i}$ has at least $m$ zero eigenvalues. This completes the proof.

The gains $L_{i}$ are retrieved by $L_{i}=T_{i}^{c} \tilde{L}_{i}$. The constraint on the structure of $\tilde{L}_{i}$ ensures (15) to be input independent by applying Proposition 1.

Now, the block matrix $\tilde{K}_{i}^{1}$ of $\tilde{L}_{i}$ must be computed in order to obtain the global convergence of (16) by a suitable 
assignment of the $n-m$ remaining eigenvalues. Combined with the input independence property, (15) will have the IIGS property.

\section{B. IIGS conditions}

In this subsection, the result ensuring the IIGS property is stated.

Let some matrices $\tilde{G}_{i}$ get the form :

$$
\tilde{G}_{i}=\left[\begin{array}{cc}
\tilde{G}_{i}^{11} & \mathbf{0} \\
\mathbf{0} & \tilde{G}_{i}^{22}
\end{array}\right]
$$

Besides, consider the partitions of some matrices $\tilde{S}_{i}$ :

$$
\tilde{S}_{i}=\left[\begin{array}{cc}
\tilde{S}_{i}^{11} & \tilde{S}_{i}^{12} \\
\tilde{S}_{i}^{21} & \tilde{S}_{i}^{22}
\end{array}\right]
$$

Theorem 2. The drive-response system (1-2) is IIGS if there exist symmetric positive definite matrices $\tilde{S}_{i}, \tilde{G}_{i}$ partitioned as above and matrices $\tilde{F}_{i}$ of appropriate dimensions such that

$$
\left[\begin{array}{cccc}
\tilde{G}_{i}^{11}+\left(\tilde{G}_{i}^{11}\right)^{T}-\tilde{S}_{i}^{11} & (\bullet)^{T} & (\bullet)^{T} & (\bullet)^{T} \\
-\tilde{S}_{i}^{21} & \tilde{G}_{i}^{22}+\left(\tilde{G}_{i}^{22}\right)^{T}-\tilde{S}_{i}^{22} & (\bullet)^{T} & (\bullet)^{T} \\
\left(\tilde{A}_{i}^{11}\right)^{T} G_{i}^{11}-\left(\tilde{A}_{i}^{21}\right)^{T} \tilde{F}_{i} & \left(\tilde{A}_{i}^{21}\right)^{T} \tilde{G}_{i}^{22} & \tilde{S}_{j}^{11} & (\bullet)^{T} \\
0 & 0 & \tilde{S}_{j}^{21} & \tilde{S}_{j}^{22}
\end{array}\right]>0
$$

The gains $\tilde{K}_{i}^{1}$ satisfy $\tilde{K}_{i}^{1}=\left(\tilde{G}_{i}^{11}\right)^{-T} \tilde{F}_{i}^{T}$.

Proof Let $\tilde{A}_{i}$ be the matrices obtained from $A_{i}$ under the piecewise linear similarity transform fulfilling (18). Furthermore, let $\tilde{L}_{i}$ be the gain matrices of which structure is defined as in Proposition 2. In this proposition, it has been shown in subsection III-A that such gains make the equation (16) of the error to be input independent. Besides, global convergence of (16) is equivalent to global convergence of (17) according to the result of section II-B. And yet, global convergence of (17) is also equivalent to global convergence of the dual system :

$$
\tilde{\nu}_{k+1}=\left(\tilde{A}_{i}-\tilde{L}_{i} \tilde{C}_{i}\right)^{T} \tilde{\nu}_{k}
$$

Substitute $A_{i}$ by $\left(\tilde{A}_{i}-\tilde{L}_{i} \tilde{C}_{i}\right)^{T}, G_{i}$ by $\tilde{G}_{i}$ and $S_{i}$ by $\tilde{S}_{i}$ in (11). Finally, making use to the change of variable $\tilde{F}_{i}=\left(\tilde{K}_{i}^{1}\right)^{T} \tilde{G}_{i}^{11},(21)$ is obtained. The Matrix Inequalities of Theorem 2 ensure (22) and so (16) to be poly-quadratic stable, which is sufficient for global asymptotical stability. Combined with the input independence property, (3) is fulfilled 
Remark 2. It can be easily shown that the special diagonal structure of the matrices $\tilde{G}_{i}$ causes the problem to be solvable as a set of Linear Matrix Inequalities while a non diagonal structure would have lead to Bilinear Matrix Inequalities constraints. Consequently, such a matrix allows not only the linearity of the constraints (and so the convexity of the problem) but also prevents a restriction on the structure of $\tilde{S}_{i}$ involved in the PDLF, which would have rendered the problem to be more conservative. Indeed, let us recall that quadratic stability is a special case of poly-quadratic stability when taking $\tilde{G}_{i}=\tilde{S}_{i}=\tilde{S}^{*}$.

Remark 3. The global stability is ensured since all the switches are taken into account, regardless of the rule which orchestrates them. As a consequence, the theorem holds regardless of the chaotic motion exhibited by the drive. If the detailed study of the chaotic dynamics reveals that some specific switches cannot occur, the conservatism can be reduced by removing the corresponding pairs $(i, j)$ in (21).

\section{General Design Procedure}

A general procedure for designing an input independent global synchronization of a drive-response of the form (1-2), that is for computing the pairs $\left(M_{i}, L_{i}\right)$, given $A_{i}, E_{i}$ and $C_{i}$, consists in the following points.

i) perform $T_{i}^{c}$ such that $C_{i} T_{i}^{c}=\left[\begin{array}{ll}\mathbf{0} & \mathbf{1}\end{array}\right]$

ii) compute $\tilde{K}_{i}^{1}=\left(\tilde{G}_{i}^{11}\right)^{-T} \tilde{F}_{i}^{T}$ with $\tilde{G}_{i}^{11}$ and $\tilde{F}_{i}$ solutions of (21)

iii) compute successively $\tilde{L}_{i}=\tilde{A}_{i} \tilde{K}_{i}$ with $\tilde{K}_{i}=\left[\begin{array}{ll}\tilde{K}_{i}^{1} & \mathbf{1}\end{array}\right]^{T}$ and $L_{i}=T_{i}^{c} \tilde{L}_{i}$

iv) set $M_{i}=N_{i}$ with $N_{i}$ the matrix whose columns span the null space of $A_{i}-L_{i} C_{i}$

It is recalled that IIGS allows the synchronization regardless to $u_{k}$. Now, the following proposition provides a way to recover $u_{k}$ since it is not directly available on the response part.

Proposition 4 Provided that $\operatorname{rank}\left(C_{i} N_{i}\right)=m$, the quantity $\hat{u}_{k}$ defined as :

$$
\hat{u}_{k}=\left(C_{i} N_{i}\right)^{-1}\left(y_{k}-C_{i} \hat{x}_{k}\right)
$$

will converge towards $u_{k}$. 
Proof From (23) and the output equation of (1) with $M_{i}=N_{i}$, one obtains :

$$
u_{k}-\hat{u}_{k}=-\left(C_{i} N_{i}\right)^{-1} C_{i}\left(x_{k}-\hat{x}_{k}\right)
$$

Thus, global convergence of $\hat{x}_{k}$ towards $x_{k}$ implies global convergence of $\hat{u}_{k}$ towards $u_{k}$.

\section{Illustrative EXAmples}

To illustrate the results above, consider the map of which state space representation of the drive and the response is respectively of the form (1) and (2) with:

- $x_{k}=\left[\begin{array}{lll}x_{k}^{1} & x_{k}^{2} & x_{k}^{3}\end{array}\right]^{T}$ and $\hat{x}_{k}=\left[\begin{array}{ccc}\hat{x}_{k}^{1} & \hat{x}_{k}^{2} & \hat{x}_{k}^{3}\end{array}\right]^{T}$
- $A_{i}=\left[\begin{array}{ccc}0 & 0.4 & 1 \\ h_{i} & 0.4 & 0 \\ -0.8 & 0 & 0.9\end{array}\right], h_{1}=-1.12, h_{2}=1$
- $E_{1}=\left[\begin{array}{lll}0 & 0 & 0\end{array}\right]^{T}$ and $E_{2}=\left[\begin{array}{lll}0 & -12.72 & 0\end{array}\right]^{T}$

- the two regions respectively associated to $A_{1}$ and $A_{2}$ are $R_{1}$ and $R_{2} . R_{1}$ is the set $\left\{x_{k} \mid x_{k}^{1}<6\right\}$ and $R_{2}$ is the set $\left\{x_{k} \mid x_{k}^{1} \geq 6\right\}$

- the output matrix is chosen to be a constant matrix : $C=\left[\begin{array}{lll}0 & 0 & 1\end{array}\right]$

This map exhibits a chaotic motion. This complex motion is used in order to mask an information $u_{k}$ through an embedding corresponding to (2). Let us apply the design procedure of subsection III-C to ensure the recovering of $u_{k}$.

\section{$\underline{\text { step } i)}$}

This step is useless since $T_{i}^{c}=\mathbf{1}$ as the output matrix $C$ is already in the canonical form.

\section{step $i i)$}

Since $T_{i}^{c}$ equals the identity matrix, $\tilde{A}_{i}=A_{i}$. The set of Linear Matrix Inequalities (21) are solved by using a LMI tool (see for instance [18]). The resulting unknown matrices involved in the gains computation are :

$$
\begin{aligned}
& -\tilde{G}_{1}^{11}=\tilde{G}_{2}^{11}=0.6938 \\
& \text { - } \tilde{F}_{1}=\left[\begin{array}{ll}
-0.0514 & 1.2837
\end{array}\right] \\
& \text { - } \tilde{F}_{2}=\left[\begin{array}{ll}
0.0459 & -1.1462
\end{array}\right]
\end{aligned}
$$


The corresponding gains $\tilde{K}_{i}^{1}=\left(\tilde{G}_{i}^{11}\right)^{-T} \tilde{F}_{i}^{T}$ are $\tilde{K}_{1}^{1}=\left[\begin{array}{ll}-0.0741 & 1.8503\end{array}\right]^{T}$ and $\tilde{K}_{2}^{1}=\left[\begin{array}{ll}0.0661 & -1.6520\end{array}\right]^{T}$.

$\underline{\text { step } i i i)}$

The gains of the observer $L_{i}=\tilde{L}_{i}=\tilde{A}_{i} \tilde{K}_{i}$ are computed which yields $L_{1}=\left[\begin{array}{lll}1.7401 & 0.8231 & 0.9593\end{array}\right]^{T}$ and $L_{2}=\left[\begin{array}{lll}0.3392- & -\end{array}\right.$ $0.59470 .8471]^{T}$.

\section{$\underline{\text { step iv) }}$}

Both vectors spanning the one-dimensional null space of $A_{i}-L_{i} C_{i}(m=1)$ associated to each region $R_{i}$ are computed. We get $N_{1}=\left[\begin{array}{lll}0.0352 & -0.8792 & -0.4752\end{array}\right]^{T}$ and $N_{2}=\left[\begin{array}{lll}0.0342 & -0.8550 & 0.5175\end{array}\right]^{T}$. Then, we set $M_{i}=N_{i}$.

To assess IIGS, based upon the resulting pairs $\left(M_{i}, L_{i}\right)$, the proposed drive-response setup has been designed such that the sinusoidal signal depicted on Fig $1 \mathrm{~A}$ is masked by $x_{k}$ of the chaotic map and recovered after synchronization.

Results are reported on Fig 1BCD. Before the discrete time $k=60$, the gains of the observer are set to zero such that the drive and the response are not synchronized. On Fig $1 \mathrm{~B}$, it can be seen that before $k=60$, the recovered signal is completely wrong. After $k=60$ and a synchronization transient, the information $u_{k}$ is successfully retrieved. Fig 1C and Fig 1D correspond respectively to the state reconstruction error $\left\|x_{k}-\hat{x}_{k}\right\|$ and the difference $u_{k}-\hat{u}_{k}$ between the recovered information and the initial one. Clearly, both errors converge towards zero.

Fig. 1. A : information $u_{k}$. B : recovered information $\hat{u}_{k}$. C : state reconstruction error $\left\|x_{k}-\hat{x}_{k}\right\| . \mathrm{D}: u_{k}-\hat{u}_{k}$

\section{Conclusion}

Input independent global chaos synchronization of switched systems has been examined. The synchronization is achieved based upon a response system getting an observer structure. First, a suitable partial pole placement obtained by the choice of a special structure of the observer gains ensures a partial zero eigenvalues assignment and turns the equation governing the error of reconstruction to be independent from the input. Secondly, poly-quadratic stability, more general than quadratic stability, ensures the global convergence while preventing conservatism. The drive-response system is referred to as Input Independent Global Synchronized. A preliminary practical implementation of the scheme described in this note has shown the possible interest of the IIGS concept in a secured communication context [19] even though it deserves further works to obtain a viable encryption scheme. From a theoretical point of view, it is 
worth pointing out that the problem treated here differs from the unknown input observer approach for piecewise linear systems investigated in [20][21] where the involved systems have relative degree equal to one.

\section{REFERENCES}

[1] Pecora L. M. and Carroll T. L. Synchronization in chaotic systems. Phys. Rev. Lett., 64:821-824, 1990.

[2] Nijmeijer H. and Mareels I. M. Y. An observer looks at synchronization. IEEE Trans. Circuits. Syst. I: Fundamental Theo. Appl, 44:882-890, October 1997.

[3] Huijberts H. J. C., Lilge T., and Nijmeijer H. Nonlinear discrete-time synchronization via extended observers. International Journal of Bifurcation and Chaos, 11(7):1997-2006, 2001.

[4] Pogromsky A. and Nijmeijer H. Observer-based robust synchronization of dynamical systems. International Journal of Bifurcation and Chaos, 8(11):2243-2254, 1998.

[5] Sira ramirez H. and Cruz hernandez C. Synchronization of chaotic systems :a generalized hamiltonian approach. International Journal of Bifurcation and Chaos, 11(5):1381-1395, 2001.

[6] G. Millerioux and J. Daafouz. Polytopic observer for global synchronization of systems with output measurable nonlinearities. International Journal of Bifurcation and Chaos, 13(3):703-712, March 2003.

[7] Special Issue. Control of chaos and synchronization. Syst. Control Letters, 31:259-322, 1997.

[8] Special Issue. Chaos synchronization and control : theory and applications. IEEE Trans. Circuits. Syst. I: Fundamental Theo. Appl, 40:853-1039, 1997.

[9] Special Issue. Control and synchronization of chaos. International Journal of Bifurcation and Chaos, $10(4), 2000$.

[10] I.I. Blekhman E. Mosekilde, A.L. Fradkov, editor. Special Issue on Chaos Synchronization and Control, volume 58. elsevier, 2002.

[11] L. Kocarev. Chaos-based cryptography :a brief overview. IEEE Circuits and Systems Magazine, 1(3):6-21, 2001.

[12] Huijberts H. J. C., Nijmeijer H., and Willems R. System identification in communication with chaotic systems. IEEE Trans. Circuits. Syst. I: Fundamental Theo. Appl, 47(6):800-808, 2000.

[13] A. L. Fradkov and A. Y. Markov. Adaptive synchronization of chaotic systems based on speed-gradient method and passification. IEEE Trans. Circuits. Syst. I: Fundamental Theo. Appl, 44(10):905-912, Oct. 1997.

[14] M. T. Soylemez and N. Muro. A parametric solution to the pole assignment problem using dynamic output-feedback. IEEE Trans. on Automatic Control, 46(5):711-723, May 2001.

[15] K. Sugimoto. Partial pole placement by lq regulators :an inverse problem approach. IEEE Trans. on Automatic Control, 43(5):1769-1776, May 1998.

[16] J. Daafouz and J. Bernussou. Parameter dependent lyapunov functions for discrete time systems with time varying parametric uncertainties. Systems and Control Letters, 43:355-359, 2001.

[17] J. Daafouz and G. Millerioux. Poly-quadratic stability and global chaos synchronization of discrete time hybrid systems. Special Issue of Mathematics and Computers in Simulation, 58:295-307, March 2002.

[18] M. C. de Oliveira, D.P. Farias, and J.C. Geromel. LMI solver. http://www.dt.fee.unicamp.br/ carvalho/software.html.

[19] G. Millerioux, G. Bloch, J. M. Amigo, A. Bastos, and F. Anstett. Real-time video communication secured by a chaotic key stream cipher. In Proc. of IEEE 16th European Conference on Circuits Theory and Design, ECCTD'03, pages 245-248, Krakow, Poland, September 1-4 2003.

[20] G. Millerioux and J. Daafouz. Unknown input observers for message-embedded chaos synchronization of discrete-time systems. International Journal of Bifurcation and Chaos, 14(4), April 2004. 
[21] G. Millerioux and J. Daafouz. Unknown input observers for switched linear discrete time systems. In Proc. of the American Control Conference ACC'2004, Boston Sheraton Hotel, MA, June 30 - July 22004. 